\title{
Comparative study of dimer-vacancies and dimer-vacancy lines on $\mathrm{Si}(001)$ and $\mathrm{Ge}(001)$
}

\author{
Cristian V. Ciobanu, Dhananjay T. Tambe and Vivek B. Shenoy \\ Division of Engineering, Brown University, Providence, RI 02912
}

October 19, 2018

\begin{abstract}
Although the clean $\mathrm{Si}(001)$ and $\mathrm{Ge}(001)$ surfaces are very similar, experiments to date have shown that dimer-vacancy (DV) defects self-organize into vacancy lines (VLs) on $\mathrm{Si}(001)$, but not on $\mathrm{Ge}(001)$. In this paper, we perform empiricalpotential calculations aimed at understanding the differences between the vacancies on $\mathrm{Si}(001)$ and $\mathrm{Ge}(001)$. We identify three energetic parameters which characterize the DVs on the two surfaces: the formation energy of single DVs, the attraction between two DVs in adjacent dimer rows, and the strain sensitivity of the formation energy of DVs and VLs. At the empirical level of treatment of the atomic interactions (Tersoff potentials), all three parameters are favorable for the self-assembly of DVs on the $\mathrm{Si}(001)$ surface, but not on $\mathrm{Ge}(001)$. The most significant difference between the defects on $\mathrm{Si}(001)$ and on $\mathrm{Ge}(001)$ concerns the formation energy of single DVs, which is three times larger in the latter case. By calculating the straindependent formation energies of DVs and VLs, we propose that the experimental observation of self-assembly of vacancies on clean $\mathrm{Ge}(001)$ could be achieved by applying compressive strains of the order of $2 \%$.
\end{abstract}

Keywords: Surface defects, Self-assembly, Surface energy, Semi-empirical models and model calculations, Silicon, Germanium

${ }^{*}$ Corresponding author. Email: ciobanu@engin.brown.edu 


\section{Introduction}

Epitaxial growth of germanium on the $\mathrm{Si}(001)$ surface has been an active area of investigation because it represents a versatile way of fabricating quantum dots. At the same time, $\mathrm{Ge} / \mathrm{Si}(001)$ provides an example of a system where the mismatch strain can be used to control the morphological features of the surface. In the early stages of growth, the surface changes its reconstruction from the $2 \times 1$ structure to a $2 \times N$ pattern, which consists of an array of long, parallel lines of dimer vacancies. It has recently been shown that the $2 \times N$ reconstruction can be an excellent template for the nanofabrication of 1-dimensional structures with unique electronic transport behavior 11, 2, 3. At later stages of growth, the film breaks up into pyramidal islands (quantum dots); controlling the size, spatial distribution and electronic properties of these quantum dots represent key issues for the practical applications of the heteroepitaxial growth on $\mathrm{Si}(001)$. From a fundamental point of view, the current state-of-the-art in scanning tunnelling microscopy has helped renew the general interest in the $2 \times N$ structures on $\operatorname{Si}(001)$. For example, Rastelli and coworkers [4] have recently discovered that the $2 \times N$ reconstruction on the Ge-covered $\mathrm{Si}(001)$ surface disappears upon Si capping, confirming in an ingenious way the role played by the mismatch strain in determining the stability of the vacancy lines. In another recent study [5], Sutter et al. have used high-resolution STM imaging in conjunction with a detailed statistical analysis of morphological features of the surface to establish that the interactions between steps and vacancy lines cause the initial surface roughening of the $\mathrm{Ge} / \mathrm{Si}(001)$.

Rapid progress has been made after the discovery of the $2 \times N$ structure [6, [], and two authoritative reviews summarizing the present understanding of the growth of germanium films on $\mathrm{Si}(001)$ have been published [8, 9]. Dimer vacancies (DVs) form easily on the $\mathrm{Si}(001)$ surface, but they only organize into vacancy lines (VLs) when there is a sufficient amount of Germanium deposited (close to $1 \mathrm{ML}$ ). The mismatch strain created by epitaxial deposition increases the number of dimer vacancies per unit area, and the interactions between these vacancies become important at sufficiently high vacancy concentrations. The interaction between two DVs is long-range repulsive when the DV's belong to the same dimer row, but weakly attractive when they belong to adjacent dimer rows [10, 11. Such anisotropy of the interactions determines the alignment of the DVs in the direction perpendicular to the dimer rows. This physical picture for the origin of the $2 \times N$ reconstruction has emerged from the experimental work of Chen et al. [10. The mechanism for the formation of vacancy lines is consistent not only with experiments done on other epitaxial systems (e.g., $\mathrm{Bi} / \mathrm{Si}(001)$ [12]), but also with the observations of the $\mathrm{Si}(001)$ surface with metal impurities such as $\mathrm{Ag}$ [13] or Ni 14, 15].

While the physical origin of the vacancy lines on $\mathrm{Si}(001)$ has been elucidated [10, it remains a puzzle as to why vacancy lines are not normally observed on the very similar $\mathrm{Ge}(001)$ surface. The fact that the $\mathrm{Ge}(001)$ surface is virtually defect free [16, 17] suggests that the formation energy of the vacancies on $\mathrm{Ge}(001)$ is substantially larger than that of the vacancies on $\mathrm{Si}(001)$. Quantitative data from atomic-scale calculations on the Ge system is 
not available, although the need for such calculations was emphasized almost a decade ago in the work of Yang et al. [18. The purpose of our paper is to address the differences between $\mathrm{Ge}(001)$ and $\mathrm{Si}(001)$ concerning the formation of dimer-vacancies and their self-assembly into vacancy lines. The work presented here fits well into the current efforts [17 for explaining the differences between the two technologically important surfaces.

We have calculated the formation energies of point and line defects on the (001) surfaces and their dependence on applied strain, using empirical potential [19, 20] models for interactions. The results obtained offer an explanation for the differences between the vacancies on $\mathrm{Si}(001)$ and $\mathrm{Ge}(001)$ and are consistent with experimental findings; moreover, these results could foster further work based on more accurate descriptions of the atomic interactions such as tight-binding or density functionals. We have considered both the heteroepitaxial system $\mathrm{Ge} / \mathrm{Si}(001)$ for different values of Ge coverage, as well as pure Si and Ge surfaces under external strain. While the results concerning the epitaxial $\mathrm{Ge} / \mathrm{Si}(001)$ systems have been previously obtained in different forms 21, 22, 23, the variation of the energetic parameters of DVs and VLs on the (001) surface with applied strains has not been systematically addressed in the past. Such a study of the energetics of defects on (001) surfaces as a function of applied strain is presented here. The new results obtained in this paper are:

(a) the formation energy of the dimer-vacancies on $\mathrm{Ge}(001)$ and its decrease under compressive, biaxial strains. In the case of unstrained surfaces, we have found that the formation energy for single $\mathrm{DV}$ on $\mathrm{Ge}(001)$ is three times larger than the corresponding value for the single DV on $\mathrm{Si}(001)$. Therefore, in order for the formation energy of a single-DV on Ge(001) to become negative, a large compressive strain should be applied, either biaxially or in the direction of the dimer rows.

(b) the formation energy of vacancy lines on $\mathrm{Si}(001)$ and $\mathrm{Ge}(001)$ as a function of applied strain. Using a simple model based on the interactions of nearest-neighbor vacancies in a VL, we have also identified the formation energy of a single DV and the attractive interaction (binding) between two DVs in adjacent dimer-rows as the dominant contributions to the formation energy of a VL.

The organization of this article is as follows. In section 2 we outline the competition between the formation energy of the vacancy lines and their elastic interactions as the general energetic mechanism that leads to the appearance of the $2 \times N$ reconstructions. In contrast to previous interpretations [8], we have found that there is no long range attraction between the vacancy lines, as their interactions are purely repulsive. As in most other studies to date, the formation of vacancy lines is regarded here as a purely thermodynamic phenomenon, thus the kinetics concerning the diffusion of vacancies during their alignment are not considered. In section 3 we describe the computational details of the total energy calculations based on Tersoff potentials [19, 20]. The next two sections are devoted to the formation energies and repulsion strengths for single-vacancy lines (VLs) on Ge-covered $\mathrm{Si}(001)$ (section 4) and for different types of VLs on pure $\mathrm{Si}(001)$ and $\mathrm{Ge}(001)$ (section 5). We investigate the formation energies of individual dimer vacancies on (001) surfaces and their strain dependence, identifying the 
differences between the defects on $\mathrm{Si}$ and Ge surfaces. The possible relevance of these results for future experimental work is also discussed in section 5. Our conclusions are summarized in section 6 .

\section{Energy of vacancy lines on $2 \times N$ surfaces}

The $2 \times N$ reconstruction is a structural pattern on the (001) surface of $\mathrm{Si}$, characterized by the periodic lengths $2 a$ and $N a$, where $N$ is a positive integer and $a=3.84 \AA$ is the lattice constant of the unreconstructed $\mathrm{Si}(001)$ surface. This reconstruction is obtained by eliminating every $N$-th dimer from each dimer row, such that the dimer vacancies created in this manner are in registry with one another and form straight lines perpendicular to the dimer rows. To lower the number of dangling bonds at the surface, the second-layer atoms below the dimer vacancy rebond such that they are fully coordinated, at the cost of introducing some surface stress [21, 22, 23. Depending on the preparation details of the surface, other types of atomic structures of VLs can be obtained [8, 12, 13, 14, 15, 24, 25, 26. In this section we focus on the general features of the energetics of the $2 \times N$ surface, without reference to a specific atomic structure. We start by partitioning the surface energy into a contribution from the $2 \times 1$ surface and a contribution from the vacancy lines:

$$
\gamma_{2 \times N}=\gamma_{2 \times 1}+\frac{\lambda}{L_{x}}
$$

where $\gamma_{2 \times N}$ and $\gamma_{2 \times 1}$ denote the surface energies of $2 \times N$ and $2 \times 1$, respectively, $\lambda$ is the energy per unit length of the vacancy lines, and $1 / L_{x}$ is the density of lines in a periodic array of VLs with a spacing of $L_{x} \equiv N a$. Eq. (11) defines the energy per unit length $\lambda$ of the vacancy lines as an excess energy with respect to the defect-free $2 \times 1$ surface. We note that the energy of the vacancy lines $\lambda$ includes the formation energy of the lines, as well as their elastic interactions. Guided by the early work of Marchenko and Parshin [27, we expect the interactions to be inversely proportional with the square of the separation between VLs, so that the line energy $\lambda$ can be written as

$$
\lambda=\Lambda+\frac{\pi^{2}}{6} \frac{G}{L_{x}^{2}}
$$

where $\Lambda$ is the formation energy of a VL and $G$ is the strength of the repulsion between lines. The numerical factor $\pi^{2} / 6$ arises since we treat a periodic array of line defects [28, rather than two isolated VLs. Using Eq. (2) in (11) we can write the surface energy of the $2 \times N$ reconstruction as a function of $N$ :

$$
\gamma_{2 \times N}=\gamma_{2 \times 1}+\frac{\Lambda}{N a}+\frac{\pi^{2}}{6} \frac{G}{N^{3} a^{3}}
$$

where we have used the fact that $L_{x}=N a$. 


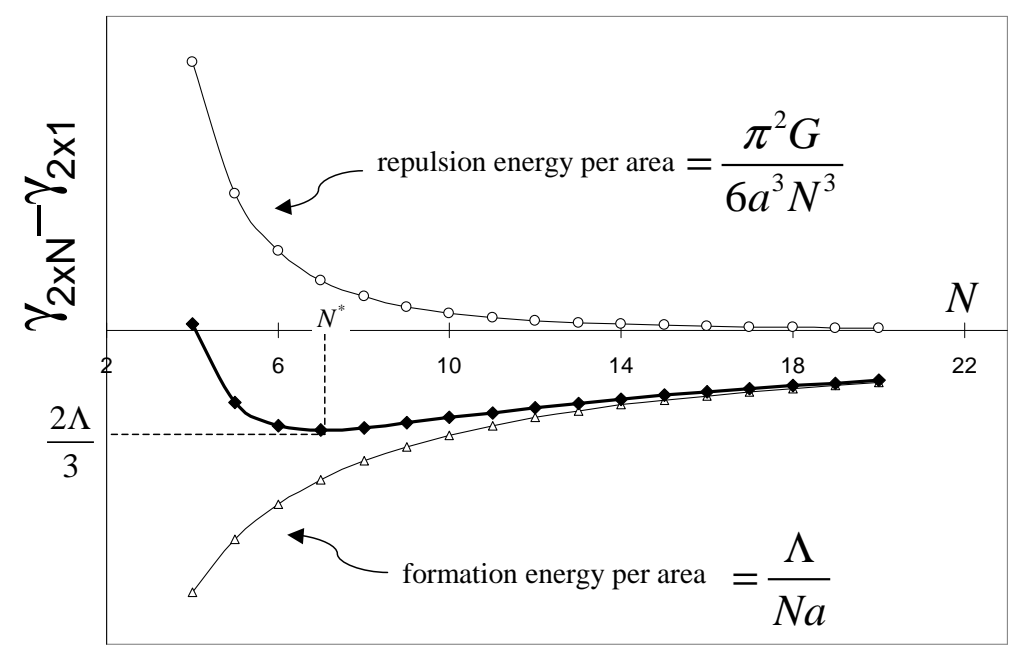

Figure 1: A typical plot of the surface energy difference $\gamma_{2 \times N}-\gamma_{2 \times 1}$ as a function of $N$ (solid diamonds). The minimum of the surface energy $\gamma_{2 \times N}$ is determined by the competition between the negative formation energy (triangles) and a repulsive (positive) interaction between the vacancy lines (circles).

Analyzing Eq. (31), we note that:

(a) if $\Lambda$ is positive then $\gamma_{2 \times N}>\gamma_{2 \times 1}$, so the $2 \times 1$ structure is energetically favorable.

(b) for any $\Lambda<0$, Eq. (3) has only one minimum located at:

$$
N=N^{*} \equiv \frac{\pi}{a} \sqrt{\frac{G}{(-2 \Lambda)}}
$$

For the case of negative formation energy $\Lambda$, a typical plot of the surface energy difference $\left(\gamma_{2 \times N}-\gamma_{2 \times 1}\right)$ as a function of $N$ is shown in Fig. 1. The condition $\Lambda<0$ is sufficient to ensure the stability of the $2 \times N$ reconstruction (over the $2 \times 1$ structure), because for $N=N^{*}$ we have $\gamma_{2 \times N}-\gamma_{2 \times 1}=(2 / 3) \Lambda<0$. This analysis of Eq. (3) shows that, irrespective of the atomic-scale features of the VLs, the $2 \times N$ reconstruction is always stable when the formation energy of a vacancy line is negative. The optimal value of $N$ is given by the competition between the formation energy $\Lambda$ and the strength $G$ of the repulsive interactions, as shown in Eq. (4). The magnitudes of $\Lambda$ and $G$ are determined by atomic-scale details, and incorporate the effects of the VL structure [21, 22], wetting layer composition [8, 23, 29], as well as the anisotropy of surface stress and relaxation [22, 23].

The following two sections are devoted to the computation of the line formation energies and repulsion strengths for various VL structures, and to the investigation of the behavior of these quantities as a function of germanium coverage and applied strain. Before we proceed 
with the numerical results, we draw a connection with previous work on the energetics of the $2 \times N$ surface. The above theory of the energetics of the vacancy lines is similar to Refs. [30, 31, since it explicitly considers the competition between the (negative) formation energy of a VL and the repulsive (positive) interaction between the VLs as the physical origin of the $2 \times N$ structure. In Ref. [30] however, the emphasis falls on the surface stress anisotropy, and the treatment of the two-domain (i.e. $2 \times N$ and $N \times 2$ ) surfaces obliterates the simplicity of the VL energetics outlined above.

A word of caution is due concerning the mechanisms of formation of the $2 \times N$ reconstruction proposed in Ref. [29] and in the review article [8]. In [29], the conclusions based on the energetic competition between "trench formation" and "strain relaxation" are fortuitous, since the $N$-dependence of each of these two energetic contributions is incorrect ${ }^{1}$. The sign of the "trench energy" and the magnitudes of the two energetic contributions are also incorrect, though these aspects are probably outside the scope of the simple Frenkel-Kontorova model used in [29]. Ref. [8] asserts that "in the dimer-row direction the VL-VL interaction is short-range repulsive and long-range attractive", which seems plausible given the behavior of the $\gamma_{2 \times N}$ in the limit of large $N$. However, we note that what appears to be an attractive long-range interaction between VLs is in fact the formation energy of one VL per unit cell of the $2 \times N$ surface. As shown in the sections to follow, the VL-VL interaction takes a purely repulsive form once the vacancy line energy is extracted from the surface energy.

\section{Computational Details}

\subsection{Structure of the vacancy lines}

In the terminology of Ref. 32, the vacancy lines considered here are made of 1-DV, 2DV and 1+2-DVs, as shown in Fig. 2. The choice of these structures is motivated by the experimental observations of the atomic-scale details of vacancy lines on different surfaces: the 1-DV structure is present on Ge-covered $\operatorname{Si}(001)$ [8, 21, 22, 23, while lines with the 2DV and 1+2-DV configurations have been observed on $\mathrm{Si}(001)$ with $\mathrm{Ni}$ [14, 15] and Ag [13] impurities, as well as on radiation-quenched $\mathrm{Si}(001)$ [24, 25].

\subsection{Total energy calculations}

Accurate calculations of the long-range behavior of the VL-VL interactions are very demanding, requiring slabs that are both long $(N>10)$ and thick (at least 10 layers). Earlier densityfunctional and tight-binding calculations were restricted to thin slabs with 4-6 moving layers, due to limitations in computational power [33, 34, 35. At present, density-functional and tight-binding calculations have become possible [36, 37, though they remain computationally expensive for the purpose of determining the long-range interactions between line defects.

\footnotetext{
${ }^{1}$ We believe that the energetic competition that determines the $2 \times N$ reconstruction is described by Eq. (3).
} 

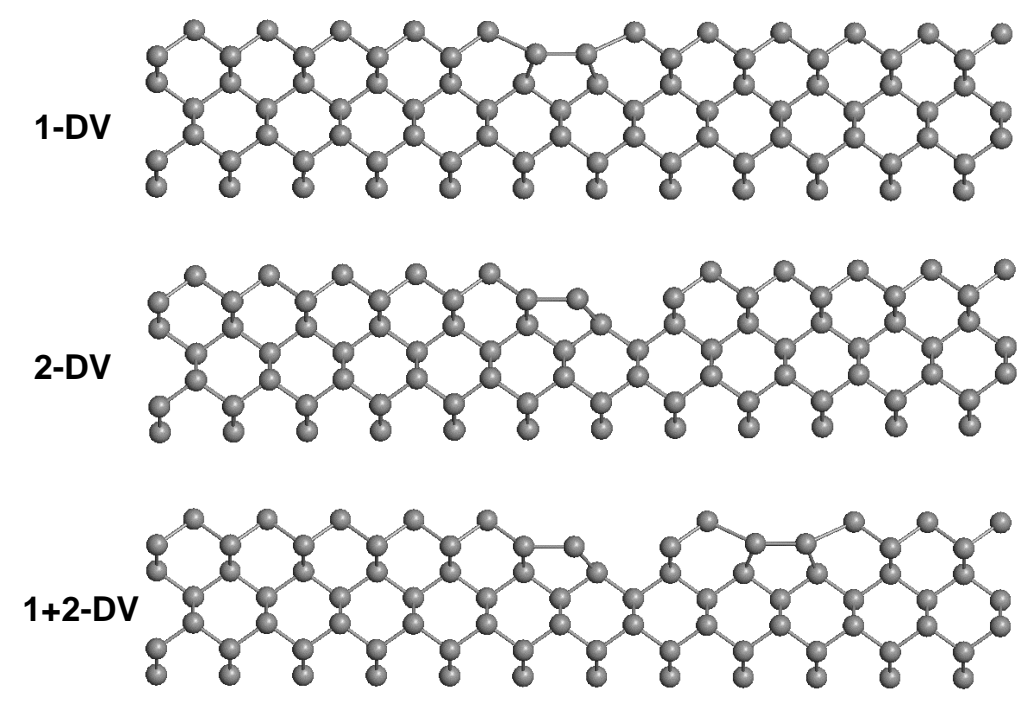

Figure 2: Atomic structures of the vacancy lines, with the nomenclature given in Ref. 32. The $1+2-\mathrm{DV}$ complex is made of a 2 -DV complex and single DV, separated by one dimer row.

Such calculations are still prohibitive for point defects on the surface, since these defects require simulation cells that are large in all three directions. As more studies would probably become available for various types of vacancy lines in the near future, it is worth pointing out that a good test for their total-energy convergence is to check that the line energies $\lambda$ can be fitted well with the inverse-square power law given by Eq. (2). Such a test would also indicate whether or not the chosen thickness of the computational slab is sufficient. This type of analysis is routinely performed for steps on crystal surfaces (see [38, 39, 40] and references therein) in order to determine the step formation energy and step-step interactions, but it has almost never been applied to the case of vacancy lines.

Since we will investigate vacancy lines as well as individual vacancies (point defects) on the surface, we employ empirical potentials to model the atomic interactions. We use the Tersoff potential for Si and Ge [19, 20, since it satisfactorily describes the structure and energetics of steps on (001) surfaces [39, 41, as well as the surface energies of the $2 \times N$ reconstructions 23]. Despite known limitations, the empirical potential described in [20] represents a widely accepted standard for Si-Ge systems with large numbers of atoms. Our aim is restricted to a meaningful comparison (rather than an accurate determination) of the properties of dimer vacancies on $\mathrm{Si}(001)$ and $\mathrm{Ge}(001)$. For this purpose, the use of the Tersoff potential is appropriate, and even desirable as a starting point.

For simulation cells with a thickness of $200 \AA$ and values of $N$ in the range $4 \leq N \leq 36$, 
we have performed conjugate-gradient structural optimizations. From the minimized total energy $E$ of the atoms in the simulation cell we compute the surface energy $\gamma_{2 \times N}$. When there is only one atomic species in the simulation cell (e.g., Si), the surface energy is given by:

$$
E=n e_{S i}+\gamma_{2 \times N} L_{x} L_{y}
$$

where $e_{S i}$ is the bulk cohesion energy per Si atom and $n$ denotes the number of atoms in the simulation cell that are allowed to relax. For the case of two atomic species in the simulation cell, the surface energy is given by:

$$
E=n_{G e} \mu_{G e}+n_{S i} \mu_{S i}+\gamma_{2 \times N} L_{x} L_{y},
$$

where $n_{G e}, \mu_{G e}\left(n_{S i}, \mu_{S i}\right)$ are the number of atoms and chemical potential of $\mathrm{Ge}(\mathrm{Si})$. Since we are considering deposition on $\mathrm{Si}(001)$, the chemical potential of $\mathrm{Si}$ is set to the bulk cohesion energy per atom $e_{S i}$. For low Ge coverages $(1-3 \mathrm{ML})$, the chemical potential of Ge $\left(\mu_{G e}\right)$ depends on the deposition conditions and is not known, although its value is usually set to the bulk cohesion energy of (unstrained) Ge, $e_{G e}$ [8, 23, 29, 35]. Exceptions are found in the work of Oviedo et al. [36] and $\mathrm{Li}$ et al. [37, who carefully define a reference state from which the surface energies are measured; the procedure in [36, 37] is equivalent to computing the chemical potential of Ge based on a wetting layer composition assumed to be thermodynamically stable. From the results of Refs. 36, 37] we infer that for a pure Ge wetting layer (no intermixing), setting $\mu_{G e}=e_{G e}$ is a poor approximation for monolayer and sub-monolayer coverages, but it becomes reasonable for $2 \mathrm{ML}$ and $3 \mathrm{ML}$ of Ge coverage. Though we use $\mu_{G e}=e_{G e}$ in the present work, we will also address the effects of changing the chemical potential of Ge.

\section{Vacancy lines on the Ge-covered $\mathrm{Si}(001)$ surface}

In this section, we calculate the surface energy of the $2 \times N$ surface with $0-3 \mathrm{ML}$ Ge coverage, then compute the line formation energy and the VL-VL interactions. Our results for the surface energies are very close to the ones originally obtained by Liu and Lagally [23] the deviations are due to the slightly different versions of the Tersoff potentials ${ }^{2}$. What differentiates the results of this section from those of Ref. [23] is that we pursue the determination of the line formation energy and VL-VL interaction strength, whereas Liu and Lagally address the interplay between surface stress and stoichiometry [23].

Using Eq. (11), we have computed the line energy $\lambda$ and plotted it as a function of the inverse square of the line spacing $\left(1 / L_{x}^{2}\right)$ in Fig. 3. The line energy $\lambda$ is a linear function of $1 / L_{x}^{2}$ for each integer value of the Ge coverage between 0ML and 3ML (Fig. 3). This

\footnotetext{
${ }^{2}$ The parameter $\lambda_{3}$ of the Tersoff potential given in [19] is set to zero in 20], thus modifying the original T3 parametrization for pure Si. Here, we have found it convenient to use a form of the interactions that reduces to 19 in the limit where only one atomic species is present in the simulation cell.
} 


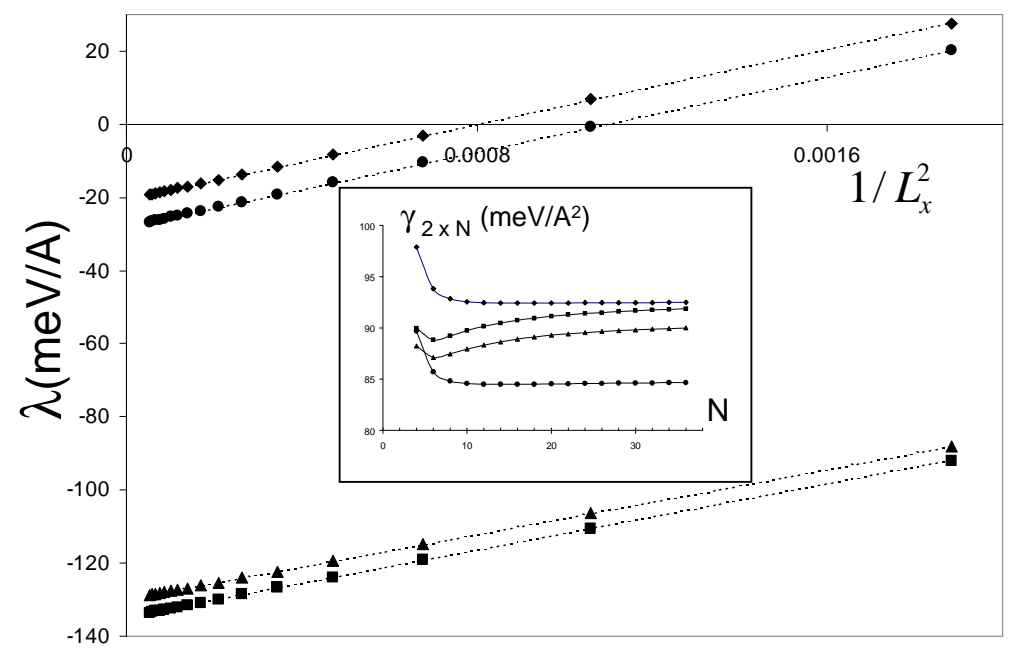

Figure 3: Vacancy line energy $\lambda$ plotted as function of inverse square of the line spacing $\left(1 / L_{x}^{2}\right)$ for different Ge coverages: $0 \mathrm{ML}$ (diamonds), $1 \mathrm{ML}$ (circles), $2 \mathrm{ML}$ (triangles) and 3 ML (squares). The inset represents the energy of the $2 \times N$ surface as a function of $N$ [23], computed with the Tersoff potential T3 [19, 20].

behavior confirms the expectation (Eq. (2) ) that the interactions between the vacancy lines are dipolar in nature [27]. The parameters of the linear fits shown in Fig. 3 give the line formation energy $\Lambda$ (intercept) and the line-line repulsion strength $G$ (slope), and are listed in Table 1. The table also includes the fitting parameters for two cases of intermixed wetting layer compositions. We have verified that the the dipolar character of the line-line interactions is not changed by intermixing in the wetting layer. These intermixing cases are considered here only for the purpose of illustration, as the actual structure of the wetting layer at given Ge coverage is under debate [4, 23, 29, 42. The data in Table 1 shows that for both of the wetting layer compositions considered (pure Ge or intermixed), the formation energy decreases strongly with increasing Ge coverage, which is consistent with previous findings (refer to [8]). The repulsion strength $G$ is very sensitive to the WL composition, as the elastic interactions are largely transmitted through the first few layers: different intermixed compositions affect the elastic properties of the film and thus the strength of the repulsive interactions.

The trends shown in Table 1]are well understood [8] and physically meaningful, although we emphasize that the exact values of the formation energy $\Lambda$ are influenced by the choice of the chemical potential for the deposited Ge atoms. By taking finite differences in Eq. (6), we note that a change $\delta \mu_{G e}$ in the chemical potential of Ge introduces a change $\delta \gamma_{2 \times N}$ in the surface energy of the $2 \times N$ structure given by:

$$
0=n_{G e}\left(\delta \mu_{G e}\right)+\left(2 N a^{2}\right)\left(\delta \gamma_{2 \times N}\right)
$$




\begin{tabular}{lccc}
\hline \hline $\mathrm{WL}$ & $\Lambda(\mathrm{meV} / \AA)$ & $G(\mathrm{eV} \AA)$ & $N^{*}$ \\
\hline $0 \mathrm{ML}$ & -20.44 & 15.562 & \\
$1 \mathrm{ML}$ & -27.95 & 15.603 & 14 \\
$2 \mathrm{ML}$ & -129.92 & 13.526 & 6 \\
$3 \mathrm{ML}$ & -134.61 & 13.818 & 6 \\
\hline $1.5 \mathrm{ML}(\mathrm{i})$ & -11.03 & 7.968 & 15 \\
$2 \quad \mathrm{ML}(\mathrm{i})$ & -12.20 & 8.541 & 15 \\
\hline \hline
\end{tabular}

Table 1: Formation energy $\Lambda$ of the vacancy lines and their repulsion strength $G$ calculated with the Tersoff potential [20] for the Ge-covered $\mathrm{Si}(001)$ surface. The first four rows correspond to a wetting layer (WL) of pure Ge 0-3 ML thick, and the last lines represent two intermixed (i) structures with the composition given in Ref. [23. The last column shows the values of $N^{*}$ obtained from Eq. (4).

where we have used the values for the periodic lengths, $L_{x}=N a$ and $L_{y}=2 a$. Similarly, for a $2 \times 1$ surface with the same area, thickness and WL composition, we have

$$
0=\left(2+n_{G e}\right)\left(\delta \mu_{G e}\right)+\left(2 N a^{2}\right)\left(\delta \gamma_{2 \times 1}\right),
$$

where the number of Ge atoms was increased by 2, as no dimers are missing from the $2 \times 1$ structure. Subtracting Eq. (8) from Eq. (77) and using Eq. (2) we obtain

$$
\delta \mu_{G e}=N a^{2}\left(\frac{\delta \Lambda}{N a}+\frac{\pi^{2}}{6} \frac{\delta G}{N^{3} a^{3}}\right),
$$

where $\delta \Lambda$ and $\delta G$ are the changes in the line formation energy and repulsion strength generated by the change in chemical potential $\delta \mu_{G e}$. From Eq. (9) in the limit of large $N$, we find that $\delta \Lambda=\delta \mu_{G e} / a$ and $\delta G \equiv 0$. Therefore, modifications of the Ge chemical potential amount to shifting the line formation energy while leaving the VL-VL interactions unchanged -a conclusion which is independent of the model used to describe the atomic interactions.

In summary, we have computed the line formation energies and the repulsion strengths for certain Ge coverages and WL compositions. As shown in Table1 the line formation energy $\Lambda$ is sensitive to the WL thickness and composition, while the VL-VL interactions are mostly sensitive to composition. To gain further insight into how strain affects the interactions and self-organization of vacancy lines, we will now consider $2 \times N$-reconstructed surfaces of pure species (either Si or Ge) under external applied strain.

\section{Defects on $\mathrm{Si}(001)$ and $\mathrm{Ge}(001)$ under applied strain}

The study of vacancy lines and dimer vacancies on uniformly strained $\mathrm{Si}(001)$ and $\mathrm{Ge}(001)$ surfaces has two technical advantages: (a) the ambiguity regarding the value of chemical 


\begin{tabular}{c|c|cc|lcc} 
& VL & $G_{0}$ & $\Lambda_{0}$ & \multicolumn{3}{c}{$\Lambda^{\prime}(\mathrm{meV} / \AA)$} \\
& structure & $\mathrm{eV \AA}$ & $\mathrm{meV} / \AA$ & $\operatorname{uniax}(x)$ & $\operatorname{uniax}(y)$ & equibiax. \\
\hline \hline \multirow{3}{*}{$\mathrm{Si}$} & $1-\mathrm{DV}$ & 15.56 & -20.56 & 4868.5 & -412.55 & 4468.5 \\
& $2-\mathrm{DV}$ & 5.01 & 51.57 & 2879.3 & -822.08 & 2067.7 \\
& $1+2-\mathrm{DV}$ & 33.33 & 30.56 & 6530.6 & -1228.5 & 5354.2 \\
\hline \multirow{3}{*}{$\mathrm{Ge}$} & $1-\mathrm{DV}$ & 11.12 & 32.48 & 4112.0 & -559.72 & 3559.3 \\
& $2-\mathrm{DV}$ & 4.00 & 69.613 & 2543.1 & -846.11 & 1709.6 \\
& $1+2-\mathrm{DV}$ & 24.36 & 104.64 & 5617.1 & -1396.5 & 4230.8 \\
\hline \hline
\end{tabular}

Table 2: Repulsion strength $G_{0}$ and line formation energy $\Lambda_{0}$ at zero strain for the vacancy line structures shown in Fig. 2. The last three columns show the strain sensitivity $\Lambda^{\prime}$ of the line formation energy for different types of in-plane deformation. The results in this table are obtained using the Tersoff potentials [19, 20] .

potential of Ge is removed, and (b) the uncertainties related to intermixing in the wetting layer obviously do not arise when studying systems with only one atomic species. Therefore, the study of defects on clean surfaces under external strain allows for a more direct comparison between $\mathrm{Si}(001)$ and $\mathrm{Ge}(001)$.

\subsection{Vacancy lines on strained surfaces}

The surface energy of the $2 \times N$ structure under external strain can be computed using formula (5) in which the bulk cohesion energy is also a function of the applied strain. The in-plane deformations are applied along the dimer rows $\left(\epsilon_{x} \equiv \varepsilon, \epsilon_{y}=0\right)$, perpendicular to the dimer rows $\left(\epsilon_{x}=0, \epsilon_{y} \equiv \varepsilon\right)$, or equibiaxially $\left(\epsilon_{x}=\epsilon_{y} \equiv \varepsilon\right)$. For each type of deformation we vary the strain in the range $-2 \% \leq \epsilon \leq 2 \%$, by appropriately scaling the atomic coordinates. In this range of deformation, we find that the line formation energy $\Lambda$ is a linear function of strain:

$$
\Lambda\left(\epsilon_{x}, \epsilon_{y}\right)=\Lambda_{0}+\Lambda_{\alpha}^{\prime} \varepsilon
$$

where $\Lambda_{0}$ is the line formation energy in the absence of strain, and the subscript $\alpha$ denotes the uniaxial strain along different directions $(\alpha=x$, or $\alpha=y)$ or the equibiaxial strain $(\alpha=x y)$. A consequence of the linear relation described in Eq. (10) is that the biaxial strain sensitivity $\Lambda_{x y}^{\prime}$ is the sum of the two sensitivities $\Lambda_{x}^{\prime}$ and $\Lambda_{y}^{\prime}$ that correspond to the uniaxial deformations:

$$
\Lambda_{x y}^{\prime}=\Lambda_{x}^{\prime}+\Lambda_{y}^{\prime}
$$

For the VL structures shown in Fig. 2] we have computed all the strain sensitivities $\Lambda_{\alpha}^{\prime}(\alpha=x, y, x y)$ independently, and found that formula (11) holds to within 1-2\% of $\Lambda_{x y}^{\prime}$. These strain sensitivities are listed in Table 2. along with the formation energies $\Lambda_{0}$ and repulsion strengths $G_{0}$ computed in the absence of strain. The strain-dependence of the 
line formation energy $\Lambda$ is strongly anisotropic. As shown in Table 2 the strain sensitivity $\Lambda_{x}^{\prime}$ along the dimer row is positive, while $\Lambda_{y}^{\prime}$ is negative and several times smaller (up to one order of magnitude for 1-DV vacancy lines) in magnitude than $\Lambda_{x}^{\prime}$. This indicates that for all the vacancy structures shown in Fig. 2, the rebonding in the second layer plays a crucial role in determining the strain dependence of the line formation energy. In the absence of strain, the bonds that "bridge" the second-layer atoms in the vacancy lines are stretched compared to their bulk values, and are especially sensitive to strains applied parallel to them. Therefore, compressive strains along the dimer rows will decrease the amount of stretch in those bonds, lowering the formation energy of the vacancy lines. This correlation between atomic bonding and formation energy is consistent with the ones given previously for the case of 1-DV lines (see, for example [8, 21, 22, 23]). On the other hand, an uniaxial strain applied perpendicular to the dimer rows only indirectly affects the formation energy of the vacancy lines via a Poisson-type effect: a stretch along the $y$-direction creates a small compressive stress in the $x$-direction, which in turn lowers the line formation energy.

While the formation energy $\Lambda$ of the vacancy lines varies significantly with applied strain, the VL-VL repulsion strength $G$ shows a weak strain dependence, changing by less than ten percent for the values of strain considered here. The reason for the weak strain-dependence of the VL interactions is that the relaxations that occur after applying external strains to an already relaxed $2 \times N$ surface are rather small, since no bonds are broken or formed. Consequently, the VL-VL interactions are not substantially affected by external strains as long as these strains remain in the small-deformation regime given by Eq. (10).

By comparing the Si and Ge data (Table 2) for each type of VL structure, we find several systematic trends: (a) the repulsion strengths $G$ are stronger for Si than for Ge, (b) the formation energies $\Lambda_{0}$ are smaller for Si than for Ge and (c) the strain sensitivities along dimer row $\left(\Lambda_{x}\right)$ and biaxial $\left(\Lambda_{x y}\right)$ are larger for Si than for Ge. Most notably, the formation energy of a single-DV line on $\mathrm{Si}(001)$ is negative, while for the case of $\mathrm{Ge}(001)$ it is positive. While there is a possibility that this sign difference between $\mathrm{Si}(001)$ and $\mathrm{Ge}(001)$ might be due to artifacts of the empirical potential, it is consistent with the observation of self assembly of DVs on $\mathrm{Si}(001)$ [26] and not on $\mathrm{Ge}(001)$ [18, 16, 43, 44]. Some of these trends are related to the formation and binding of individual dimer vacancies, as will be shown in the next section.

The occurrence of the vacancy lines is a consequence of the strongly anisotropic interactions between the individual dimer vacancies, coupled with the preference of the DVs to diffuse along the dimer rows. As discovered by Chen et al. 10, two DVs that belong to the same dimer row repel one another, while DVs in adjacent dimer rows experience a weak attraction at short distances. This finding was confirmed by other independent experiments [14, 13], as well as by theoretical studies [11. Thus the physics of vacancy lines is intrinsically related to the formation, interactions and diffusion of individual dimer-vacancies. In what follows, we show how the strain dependence of the formation energy of the VLs is linked to the behavior of individual dimer vacancies under strain. 


\subsection{Dimer vacancies on $\mathrm{Si}(001)$ and $\mathrm{Ge}(001)$}

In this section, we compute the formation energy of three types of DVs on $\mathrm{Si}(001)$ and $\mathrm{Ge}(001)$, as well as the attractive energy of two vacancies placed in adjacent dimer rows. In the case of $\mathrm{Si}(001)$, a similar calculation was performed by Wang and coworkers [32, and has served as useful reference for many subsequent investigations. In contrast, we have found no similar studies for the formation energies of defects on $\mathrm{Ge}(001)$. Here we perform such a study using the Tersoff potential [20], and identify the differences between the vacancies on the two surfaces, $\mathrm{Si}(001)$ and $\mathrm{Ge}(001)$. We denote the formation energy of a DV complex by $u$, and that of an isolated pair of complexes lying in adjacent dimer rows (called a bound pair form here on) by $u_{p}$. The binding energy of the pair is defined ${ }^{3}$ as the difference between the pair's formation energy and the formation energies of two isolated DVs:

$$
w \equiv u_{p}-2 u \text {. }
$$

We have calculated the formation and binding energies for different vacancy complexes, and have listed the results in Table 3 .

We shall first discuss the formation energy $u$, and compare our results with those of Wang et al. 32, which were obtained using density-functional calculations coupled with an empirical potential. The advantages and disadvantages of such a coupling scheme are clearly discussed in the original paper [32]. In the case of 1 -DV vacancy on $\mathrm{Si}(001)$, we find the formation energy of single DV's $u \approx 0.19 \mathrm{eV}$, in good agreement with the value of $0.22 \mathrm{eV}$ reported in Ref. 32. For the other vacancy complexes, the agreement with Ref. 32 is poor because the empirical model [19] does not accurately capture the relaxations of the undercoordinated second-layer atoms that are present in the 2 -DV and $1+2$-DV complexes. Due to this limitation, the Tersoff potential [19] predicts that the 2-DV complex is unstable with respect to the formation of two isolated single DVs, $u_{2-\mathrm{DV}}>2 u_{1-\mathrm{DV}}$. We find however that the empirical potential is still able to predict that a $2-\mathrm{DV}$ and a $1-\mathrm{DV}$ on the same dimer row can bind to form a $1+2-\mathrm{DV}$ complex (i.e. $u_{1+2 \mathrm{DV}}<u_{2-\mathrm{DV}}+u_{1-\mathrm{DV}}$ ), though the binding energy for the $1+2 \mathrm{DV}$ complex is about half the value obtained from 32 . In the case of $\mathrm{Ge}(001)$ surface, the potential [20] fares better than the silicon parametrization [19] in describing the stability of DV complexes. Both the 2 -DV and $1+2-\mathrm{DV}$ vacancies on Ge(001) are stable against breaking into isolated smaller complexes, as indicated by their respective formation energies in Table 3. This result is in agreement with the observations of Yang et al., who report that $1+2-\mathrm{DV}$ s and 2-DVs are predominant on the Ge(001) surface [18].

We now turn to a discussion of the binding energies of DV pairs $w$ given in Table 3 There are two main issues that complicate the comparison of theoretical results in Table 3 with the experimentally determined binding energies. First, we note that the binding energy of an isolated bound pair computed here and in Ref. 11] are defined in a different manner than what is usually obtained from experiments. Experiments measure the binding energy when

\footnotetext{
${ }^{3}$ With this definition, the negative sign is retained to indicate the stability of the bound pair.
} 


\begin{tabular}{|c|c|c|c|c|c|c|}
\hline & DV complex & & $(\mathrm{eV})$ & $w(\mathrm{eV})$ & $\frac{u+w}{2 a}(\mathrm{meV} / \AA)$ & $\Lambda(\mathrm{meV} / \AA)$ \\
\hline \multirow{3}{*}{ Si } & 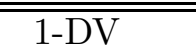 & $\overline{0.186}$ & 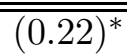 & 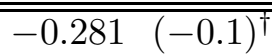 & -12.37 & -20.56 \\
\hline & $2-\mathrm{DV}$ & 0.505 & $(0.33)^{*}$ & -0.106 & 51.94 & 51.57 \\
\hline & $1+2-\mathrm{DV}$ & 0.619 & $(0.42)^{*}$ & -0.322 & 38.66 & 30.56 \\
\hline \multirow{3}{*}{$\mathrm{Ge}$} & $1-\mathrm{DV}$ & 0.549 & & -0.239 & 38.75 & 32.48 \\
\hline & $2-\mathrm{DV}$ & 0.699 & & -0.136 & 70.37 & 69.61 \\
\hline & $1+2-\mathrm{DV}$ & 1.218 & & -0.330 & 110.00 & 104.64 \\
\hline
\end{tabular}

* Ref. 32 ]

$\dagger$ Ref. 11

Table 3: Formation energies $u$ of vacancy complexes and binding energies $w$ corresponding to a bound pair of vacancy complexes situated in adjacent dimer rows. The last two columns give the line formation energies estimated using formula (13), as well as the ones obtained from total energy calculations (Table 2). The results of Wang et al. 32 and Weakliem et al. 11. for the case of silicon are indicated in parentheses.

the DVs are part of a vacancy line, while calculations address an isolated pair of vacancies. We have estimated that the binding of two vacancies (on an average) within a VL is up to $\approx 40 \%$ stronger than the binding within an isolated bound pair. Secondly, the experiments report the binding energies for DVs on $\mathrm{Si}(001)$ with a certain amount of Ge coverage [10] or metal contamination 13, 14, as opposed to clean surfaces. Given these two concerns related to experimental conditions, as well as the use of an empirical potential in this study, discrepancies between the binding energies $w$ listed in Table 3 and the ones reported experimentally are expected. However, we have found that there is reasonable agreement with the experimental data. For the single DVs on a Ge-covered $\mathrm{Si}(001)$ surface (1.5ML) Chen et al. [10] estimate the binding energy in the range $-0.18 \mathrm{eV}<w<-0.25 \mathrm{eV}$, while our result for 1-DVs on pure $\mathrm{Ge}(001)$ is $-0.24 \mathrm{eV}$ and $-0.28 \mathrm{eV}$ on pure $\mathrm{Si}(001)$ (refer to Table 3). On the Ni-contaminated $\mathrm{Si}(001)$ surfaces, Zandvliet and coworkers [14] report an average attractive interaction per unit length of $-0.11 \mathrm{eV} / a$; since the distance between two neighboring dimer rows is $2 a$, we infer that the binding energy of a pair within a $\mathrm{VL}$ is $-0.22 \mathrm{eV}$. Chang et al. report a similar figure for the binding energy $(-0.24 \mathrm{eV})$ of DVs on Ag-contaminated $\mathrm{Si}(001)$ surfaces 13 . On both $\mathrm{Ni}$ - and $\mathrm{Ag}$ - contaminated $\mathrm{Si}(001)$ the vacancy lines contain almost equal proportions of 2-DVs and 1+2-DVs [15, 14, 13] and we note that the binding energies reported in [14, 13. lie between the calculated (Table 3 ) binding energy of a 2 -DV pair $(\approx-0.11 \mathrm{eV})$ and that of an $1+2-\mathrm{DV}$ pair $(\approx-0.32 \mathrm{eV})$ on $\mathrm{Si}(001)$.

The vacancy formation energy $u$ of a vacancy complex and the binding energy $w$ of a pair determine the formation energy of the vacancy lines to a large extent. Chen et al. 10, and Weakliem et al. [1] have found that interactions between single DVs that belong to different dimer rows which are not adjacent can be neglected. This approximation implies 
that the DVs that make up a vacancy line can be treated as a 1-dimensional system with nearest-neighbor interactions. For such a system, the formation energy per unit length can be written as:

$$
\Lambda \approx \frac{u+w}{2 a},
$$

where $2 a$ is the distance between two DVs in a straight vacancy line. The data in Table 3 shows that the formation energies given by Eq. (13) are in good agreement with the line formation energies obtained from the $2 \times N$ surface energies (Table 2).

The differences between the line formation energies shown in the last two columns of Table 3 are due to the presence of interactions between a given vacancy with higher-order neighbors in the same VL, which are neglected in formula (13). While such effects could be considered small, note from Table 3 that they systematically lead to a lowering of the formation energy of vacancy lines below the estimate given by (13). As seen in the next section, the DVs are more stable as part of a vacancy line than as members of a bound pair, which is due to the presence of interactions with higher-order neighbors.

\subsection{Experimental implications}

The $\mathrm{Si}(001)$ and $\mathrm{Ge}(001)$ have been investigated intensely due to their technological and fundamental importance. As noted by Zandvliet [17, 43] there are still gaps in understanding the $\mathrm{Ge}(001)$ surface in comparison to $\mathrm{Si}(001)$, despite the similarity of the two surfaces. One aspect where the two surfaces show remarkable differences is that the vacancy lines appear easier to form on $\mathrm{Si}(001)$ rather than on $\mathrm{Ge}(001)$, as illustrated by the much larger number of reported observations in the case of silicon. To our knowledge, the only observation of vacancy lines on $\mathrm{Ge}(001)$ is for the case of $\mathrm{Bi}$ deposition (1ML) 45. No vacancy lines have so far been observed on clean $\mathrm{Ge}(001)$ [16, 18, 43, 44, while two different groups report the observation of vacancy lines on the clean $\operatorname{Si}(001)$ surface [26, 24, 25].

We look for the differences between DVs on clean $\mathrm{Si}(001)$ and $\mathrm{Ge}(001)$ by comparing their formation and binding energies in the presence of strain. Focusing on single DVs, we compare the formation energy per vacancy for isolated vacancies, bound pairs and vacancy lines under biaxial strain. This comparison is performed separately for the case of $\mathrm{Si}$ and $\mathrm{Ge}$ surfaces. The results plotted in Fig. 4 which show that the formation energy per DV, to a good approximation, is a linear function of strain for strains in the range $-2 \%-2 \%$. In contrast, we have found that the binding energy $w=u_{p}-2 u$ exhibits strong nonlinear character in this strain range; the strains at which $w$ remains linear is limited to a much smaller range, $\pm 0.2 \%$. Compressive strains lower the formation energy per DV both for the case of Si and $\mathrm{Ge}$, consistent with the previous results on vacancy lines (i.e. with the positive sign of the biaxial strain sensitivities shown in Table 2. We can now identify the difference between DVs on $\mathrm{Si}(001)$ and $\mathrm{Ge}(001)$. From Table 3 note that the formation energy $u$ of a single DV is positive both for $\mathrm{Ge}(001)$ and $\mathrm{Si}(001)$, but in the case of $\mathrm{Ge}(001)$ it is about 3 times higher than that of an isolated $\mathrm{DV}$ on $\mathrm{Si}(001)$. Therefore, in the absence of any kinetic effects, 

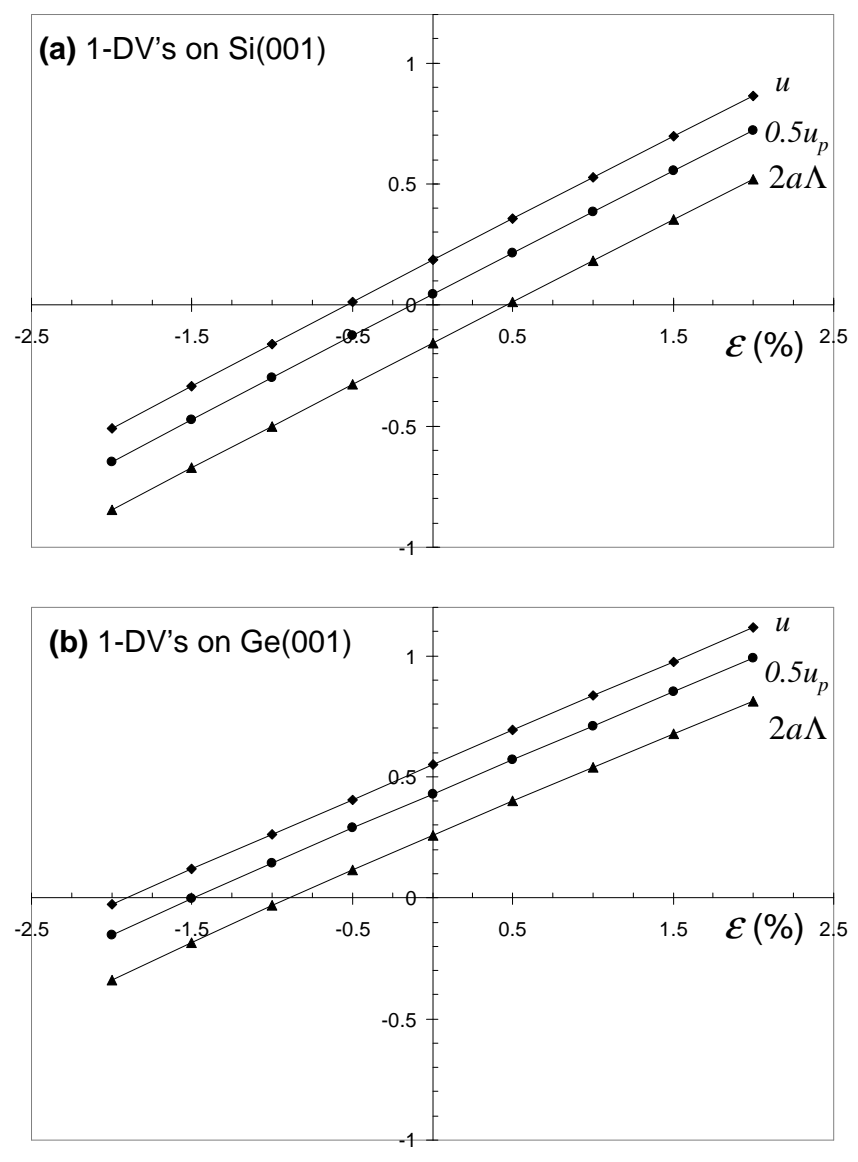

Figure 4: Formation energy $(\mathrm{eV})$ per 1-DV vacancy for an isolated DV $(u)$, a bound pair $\left(0.5 u_{p}\right)$, and a 1-DV vacancy line $(2 a \Lambda)$ as function of biaxial strain $\varepsilon$. The upper and lower figures represent the case of $\operatorname{Si}(001)$ and $\mathrm{Ge}(001)$, respectively. Note that $a$ and $\Lambda$ are the surface lattice constant and VL formation energy, both of which depend on $\varepsilon$. In each figure, the stability of DVs increases from the isolated DV to the vacancy lines, at given strain. Compressive strains lower the formation energy per DV both in the case of $\mathrm{Si}(001)$ and $\mathrm{Ge}(001)$, though in the case of Ge larger compressive strains must be applied in order to achieve negative formation energies. 
a compressive strain of $-0.5 \%$ would be required to create stable DVs $(u<0)$ on $\mathrm{Si}(001)$, which is readily achievable as result of quenching [24, 25]. In contrast, the critical value of compression needed to have single DVs on Ge(001) is four times larger, $-1.9 \%$. As seen in Fig. 4 when DVs are part of a bound pair the compression at which the formation energy per DV becomes negative decreases due to the attractive interaction between DVs: for $\mathrm{Si}(001)$ the critical compression becomes $-0.1 \%$, while for DVs on $\mathrm{Ge}(001)$ it remains rather large, $-1.5 \%$.

Based on these results (Fig. 4), we propose that organization of the DVs into vacancy lines on $\mathrm{Ge}(001)$ could be observed provided that the surface is under compressive strains of $\approx 2 \%$. Biaxial strains are somewhat difficult to apply and control accurately in practice. A simple way to provide some compression would be quenching, as done in 24, 25. As far as lowering the formation energy of DVs is concerned, uniaxial strains along the dimer row direction are more efficient than biaxial deformations. Therefore, the use of ultraflat, singledomain surfaces under uniaxial compression along the dimer row can lead to observations of vacancies organizing into lines on $\mathrm{Ge}(001)$.

\section{Conclusions}

In conclusion, we have performed an investigation of the VLs and DVs on strained $\mathrm{Si}(001)$ and $\mathrm{Ge}(001)$. We have found that the dimer-vacancies on the two surfaces exhibit differences in terms of the formation energy, their strain sensitivity, and the binding energy of two DVs in adjacent dimer rows. With the present model of atomic interactions [19, 20], each of these energetic parameters appear to favor the self-organization of DVs into vacancy lines on $\mathrm{Si}(001)$, but not on the Ge(001) surface.

The most important difference concerns the formation energy of single DVs, which was found to be $0.55 \mathrm{eV}$ for $\mathrm{Ge}(001)$, but only $0.19 \mathrm{eV}$ in the case of $\mathrm{Si}(001)$. The formation energy difference between single DVs on $\mathrm{Si}$ and Ge surfaces seems robust with respect to the empirical potential used: we have recalculated these energies with the Stillinger-Weber model [46. 47], and found similar results, though the difference between the two formation energies predicted by the Stillinger-Weber potential was larger. This finding cannot be explained by scaling the DV formation energies with the bulk cohesive energy (or melting temperature) of Si and Ge. Rather, it could likely be due to subtle details in the relaxation of the atoms that surround the vacancy, particularly to the interplay between the lateral and vertical relaxation; further studies are underway in order to elucidate this point. While it is well known that the stability of DVs on (001) surfaces is strongly enhanced by kinetic effects (i.e. two atoms are unlikely to find the DV and fill it at the same time [48]), it is conceivable that the large gap between the formation energies of DVs on $\mathrm{Si}(001)$ and $\mathrm{Ge}(001)$ determines their different physical behavior.

Based on studies of how the formation energy varies with external strains, we have

proposed that the self-assembly of vacancies on clean $\mathrm{Ge}(001)$ would be possible if compressive 
strains are applied either biaxially or along the dimer row direction. Pending an increase in the available computational power and methodologies, the results of this article warrant future studies using higher-level total-energy methods such as tight-binding or density functional

calculations. The use of such methods is expected to bring quantitative improvements of the energetic properties computed here. Furthermore, higher-level methods can also address other important aspects of the self-assembly of vacancies such as the energetic barriers associated with the creation and diffusion of $\mathrm{DVs}$ on $\mathrm{Si}(001)$ and $\mathrm{Ge}(001)$. Future investigations regarding the kinetics of dimer vacancies would be invaluable for fully elucidating the differences between $\mathrm{Si}(001)$ and $\mathrm{Ge}(001)$.

\section{Acknowledgments}

Support from the MRSEC at Brown University (DMR-0079964), National Science Foundation (grants CMS-0093714, CMS-0210095), and the Salomon Research Award from the Graduate School at Brown University is gratefully acknowledged. We thank H.J.W. Zandvliet for useful discussions on the Ni-induced vacancy lines [14, and for bringing Ref. [17] to our attention.

\section{References}

[1] J.-L. Li, X.-J. Liang, J.-F. Jia, X. Liu, J.-Z. Wang, E.-G. Wang, and Q.-K. Xue, Appl. Phys. Lett. 79, 2826 (2001).

[2] J.-Z. Wang, J.-F. Jia, X. Liu, W.-D. Chen, and Q.-K. Xue, Phys. Rev. B 65, 235303 (2002).

[3] K. Miki, J.H.G. Owen, D.R. Bowler, G.A.D. Briggs, and K. Sakamoto, Surf. Sci. 421, 397 (1999); J.H.G. Owen, K. Miki, and D.R. Bowler, Surf. Sci. 527, L177 (2003).

[4] A. Rastelli, H. von Känel, G. Albini, P. Raiteri, D.B. Migas and L. Miglio, Phys. Rev. Lett. 90, 216104 (2003).

[5] P. Sutter, I. Schick, W. Ernst, E. Sutter, Phys. Rev. Lett. 91, 176102 (2003).

[6] Y.-W. Mo and M.G. Lagally, J. Cryst. Growth 111, 876 (1991).

[7] U. Köhler, O. Jusko, B. Müller, M. Horn-von Hoegen, and M. Pook, Ultramicroscopy, 42-44, 832 (1992).

[8] F. Liu, F. Wu and M.G. Lagally, Chem. Rev. 97, 1045 (1997)

[9] B. Voigtländer, Surf. Sci. Rep. 43, 127 (2001).

[10] X. Chen, F. Wu, Z. Zhang and M.G. Lagally, Phys. Rev. Lett. 73, 850 (1994). 
[11] P.C. Weakliem, Z. Zhang and H. Metiu, Surf. Sci. 336, 303 (1995).

[12] Ch. Park, R.Z. Bakhitzin, T. Hashizume, and T. Sakurai, J. Vac. Sci. Technol. B 12, 2049 (1994).

[13] C.S. Chang, Y.M. Huang, C.C. Chen, T.T. Tsong, Surf. Sci. 367, L8 (1996).

[14] H.J.W. Zandvliet, H.K. Louwsma, P.E. Hegeman and B. Poelsema, Phys. Rev. Lett. 75, 3890 (1995).

[15] J.-Y. Koo, J.-Y. Yi,C. Hwang, D.-H. Kim, S. Lee and D.-H. Shin, Phys. Rev. B 52, 17269 (1995).

[16] S.J. Chey and D.G. Cahill, Surf. Sci. 380, 377 (1997).

[17] H.J.W. Zandvliet, Physics Reports 388, 1 (2003).

[18] W.S. Yang, X.D. Wang, K. Cho, J. Kishimoto, S. Fukatsu, T. Hashizume and T. Sakurai, Phys. Rev. B 50, 2046 (1994).

[19] J. Tersoff, Phys. Rev. B 38, 9902 (1988).

[20] J. Tersoff, Phys. Rev. B 39, 5566 (1989).

[21] K.C. Pandey, in Proceedings of the 17-th International Conference on Physics of Semiconductors, edited by D.J. Chadi and W.A. Harrison (Springer-Verlag, New York 1985) p. 55 .

[22] J. Tersoff, Phys. Rev. B 45, 8833 (1992).

[23] F. Liu and M.G. Lagally, Phys. Rev. Lett. 76, 3156 (1996).

[24] F.-K. Men, A.R. Smith, K.-J. Chao, Z. Zhang and C.-K. Shih, Phys. Rev. B 52, R8650 (1995)

[25] A.R. Smith, F.-K. Men, K.-J. Chao, Z. Zhang, and C.-K. Shih, J. Vac. Sci. Technol. B 14, 909 (1996).

[26] H. Feil, H.J.W. Zandvliet, M.H. Tsai, J.D. Dow, and I.S.T. Tsong, Phys. Rev. Lett. 69, 3076 (1992).

[27] V.I. Marchenko and A.Ya. Parshin, Sov. Phys. JETP 52, 129 (1980).

[28] R. Najafabadi and D.J. Srolovitz, Surf. Sci. 317, 221 (1994).

[29] B. Voigtländer and M. Kästner, Phys. Rev. B 60, R5121 (1999).

[30] F.-K. Men and C.-R. Hsu, Phys. Rev. B 58, 1130 (1998). 
[31] A. Natori, R. Nishiyama, and H. Yasunaga, Surf. Sci. 397, 71 (1997).

[32] J. Wang, T.A. Arias and J.D. Joannopoulos, Phys. Rev. B 47, 10497 (1993).

[33] M.-H. Tsai, Y.-S. Tsai, C.S. Chang, Y. Wei, and I.S.T. Tsong, Phys. Rev. B 56, 7435 (1997).

[34] E. Kim and C. Chen, Phys. Rev. B 66, 205418 (2002).

[35] B.D. Yu and A. Oshiyama, Phys. Rev. B 52, 8337 (1995).

[36] J. Oviedo, D.R. Bowler, and M.J. Gillan, Surf. Sci. 515, 483 (2002).

[37] K. Li, D.R. Bowler, and M.J. Gillan, Surf. Sci. 526, 356 (2003).

[38] H.C. Jeong and E.D. Williams, Surf. Sci. Rep. 34, 171 (1999).

[39] H.J.W. Zandvliet, Rev. Mod. Phys. 72, 593 (2000).

[40] M. Giesen, Prog. Surf. Sci. 68, 1 (2001).

[41] T.W. Poon, S. Yip, P.S. Ho, and F.F. Abraham, Phys. Rev. B 45, 3521 (1992).

[42] H.W. Yeom, M. Sasaki, S. Suzuki, S. Sato, S.Hosoi, M. Iwabuchi, K. Higashiyama, H. Fukutani, M. Nakamura, T. Abukawa and S. Kono, Surf. Sci. 381, L533 (1997).

[43] H.J.W. Zandvliet, B.S. Schwartzentruber, W. Wulfhekel, B.J. Hattink and B. Poelsema, Phys. Rev. B 57, R6803 (1998).

[44] T. Fukuda and T. Ogino, Appl. Phys. A 66, S969 (1998).

[45] H.K. Lowsma, H.J.W. Zandvliet, B.A.G. Kersten, J. Chesneau, A. van Silfhout and B. Poelsema, Surf. Sci. 381 L594 (1997).

[46] F.H. Stillinger and T.A. Weber, Phys. Rev. B 31, 5262 (1085).

[47] K. Ding and H.C. Andersen, Phys. Rev. B 34, 6987 (1986).

[48] Z. Zhang and H. Metiu, Phys. Rev. B 48, 8166 (1993). 